\title{
Letters
}

\section{Withdrawal of treatment in children}

SIR

The BMA medical ethics committee is consulting widely on all aspects of the withdrawing or withholding of lifeprolonging medical treatment. This openness and involvement of both lay and medical interests is welcomed.

The BMA's position is that from birth babies have the same clear rights as other patients. Where doubt or ambiguity exists about the prognosis or the child's best interests there will be a presumption in favour of preserving life unless the burdens of treatment clearly outweigh the benefits. By contrast, the Royal College of Paediatrics and Child Health (RCPCH) guidelines issued in September last year deny the newborn the same rights as adults.

There are three significant areas of concern in the $\mathrm{RCPCH}$ report that need to be highlighted. Firstly, the categorisation of children into five groups, extending the recognised clinical situations of brain death and persistent vegetative state into the undefined "no chance", "no purpose", and "unbearable" situations. The "no purpose" situation could rapidly involve eugenics. Secondly, even though it is recognised that a significant number of infants whose medical treatment is withdrawn go on to survive, the college states that it is not necessary to withdraw the paralysing agent before the respiratory support for an infant on a ventilator is withdrawn. As the presence of a paralysing agent ensures death, such practice is in essence active euthanasia. Thirdly, the concept of the "power of veto" by some senior yet undefined member of the health care team has been introduced into clinical decision making. Such powers are not recognised in the General Medical Council's "good medical practice". If, after full discussion between parents and the responsible consultant, their decision is to be vetoed by a "senior" member of the "team", it would immediately destabilise the whole concept of medical duties and responsibilities. The situation is compounded as clinical teams change each medical and nursing shift. Although the personal, religious or other views of members of the team need to be respected, to give them a power of veto over the responsible consultant raises additional medical/legal issues.

The public would be able to have more confidence in present-day paediatric practice if the Royal College of Paediatrics and Child Health had subjected its guidelines to the same open scrutiny that the BMA is currently embarking on.

JAMES APPLEYARD

Honorary Consultant Paediatrician Kent and Canterbury Hospital

\section{Best interests and persistent vegetative state}

SIR

In her thought-provoking article, Fenwick,' argues that the concept of "best interests" in a persistent vegetative state (PVS) patient is illogical, and she uses quality of life issues to justify her view. However, I would argue that a PVS patient does have "best interests" beyond quality of life issues. Furthermore, the decision about withdrawal of treatment can only be lawfully medical but is clinically irrelevant.

The problem here is the word "interests". It implies a cognitive process resulting in a preference, which by definition cannot be undertaken by a PVS patient. A better phrase would be "best options". The options available to a PVS patient are a) life and b) death. As "life" would entail continuous supportive care with no cognitive function then the difference between "life" and "death" (to the patient) would be negligible. How- $\vec{w}$ ever, to relatives this will be an appreciable difference. If PVS patients were $\overparen{\Phi}$ able to express a viewpoint then one of their "interests" might be the effect of their death or continued persistent $\omega$ vegetative state on their relatives. This takes the argument away from personalised quality of life issues to interests 0 beyond the individual but still important to him or her. Obviously, the patient is unable to express any view, and, as relatives have no legal power to $\vec{O}$ decide on treatment options, the ultimate decision as to "best interests" must lie with the medical staff.

Because all treatment for a P\&S. patient is futile (ie with no chance $\rightarrow$ fO improving prognosis or recovery) th the medical staff are being asked to deliberate between futile treatmento and no treatment. As futile treatment命 is clinically equivalent to no treatment, (and there is no legal inducement $\overrightarrow{\vec{\sigma}}$ upon the medical profession to pro- 3 vide futile treatment) it is arguable that the "withdrawal of treatment" decision is irrelevant to anything but rapidity of outcome.

I would therefore argue that a PVS patient does have interests (or options)-but these interests are in fact the interests of the relatives. This does not mean, however, that these patient interests are non-existent or invalid; simply that they have no legaD standing. Ultimately therefore the only․ㅡ. lawful decision maker is the doctor $\mathrm{N}$ and his decision is clinically irrelevant,, having no effect on outcome (death) but only on the rapidity of the outcome (weeks versus years).

\section{References}

1 Fenwick AJ. Applying best interests to persistent vegetative state - a principledO distortion? Fournal Of Medical Ethic 1998;24:86-92.

J A D STEWART, MRCI

Specialist Registrar in General Medicine anळ Gastroenterology, Department of Integrated Medicine, Glenfield General NHS Trust, Groby Road, Leicester, LE3 9QP 